\title{
Low-energy excitations in a one-dimensional orthogonal dimer model with the Dzyaloshinski-Moriya interaction
}

\author{
Kenji Sasaoka ${ }^{a}$, Chikara Ishii ${ }^{a}$, \\ ${ }^{a}$ Depertment of Physics, Tokyo University of Science, 1-3 Kagurazaka, Shinjiku-ku, Tokyo, Japan
}

\begin{abstract}
Effects of the Dzyaloshinski-Moriya (DM) interaction on low-energy excitations in a one-dimensional orthogonaldimer model are studied by using the perturbation expansions and the numerical diagonalization method. In the absence of the DM interaction, the triplet excitations show two flat spectra with three-fold degeneracy, which are labeled by magnetization $M=0, \pm 1$. These spectra split into two branches with $M=0$ and with $M= \pm 1$ by switching-on of the DM interaction and besides the curvature appears in the triplet excitations with $M= \pm 1$ more strongly than those of $M=0$.
\end{abstract}

Key words: quantum spin; low-energy excitation; Dzyaloshinski-Moriya interaction;

\section{Introduction}

Since its discovery by Kageyama et al..[1], the spin dimer compound $\mathrm{SrCu}_{2}\left(\mathrm{BO}_{3}\right)_{2}$ has attracted much attention as a suitable material for frustrated spin systems in low dimension. $\mathrm{SrCu}_{2}\left(\mathrm{BO}_{3}\right)_{2}$ exhibits various interesting phenomena, such as a quantum disordered ground state [1] and a complex shape of magnetization curve[2], because of its unique crystal structure. In consideration of the structure, Miyahara and Ueda suggested that the magnetic properties of the spin dimer compound $\mathrm{SrCu}_{2}\left(\mathrm{BO}_{3}\right)_{2}$ can be described as a spin- $1 / 2$ twodimensional (2D) orthogonal-dimer model [3], equivalent to the Shastry-Sutherland model on square lattice with some diagonal bonds [4]. The ground state of the Shastry-Sutherland model in dimer phase is exactly represented by a direct product of singlets. The low-energy dispersions possess six-fold degeneracy and are almost flat reflecting that the triplet tends to localize on vertical or horizontal bonds.

Recent experiments by ESR [5] and neutron inelastic scattering (NIS) have observed splitting of degenerate dispersions of $\mathrm{SrCu}_{2}\left(\mathrm{BO}_{3}\right)_{2}$, which can not be explained by the isotropic ShastrySutherland model. Hence Cépas et al. pointed out that the Dzyaloshinski-Moriya (DM) interaction [6] must be added between vertical and horizontal dimers in the isotropic Shastry-Sutherland model in order to explain the splitting. [7]

In this paper, as a simple model to clarify effects of the DM interaction to low-energy excitations in orthogonal-dimer systems, one-dimensional (1D) orthogonal-dimer model with the DM interaction is studied by using the perturbation theory and the numerical exact-diagonalization method. In the absence of the DM interactions, properties of 
ground state, low-energy excitations, and magnetization processes of the $1 \mathrm{D}$ orthogonal dimer model has been studied by several authors. $[8,9,10]$

\section{Model}

The Hamiltonian of the 1D orthogonal-dimer model with the DM interaction is given by

$\mathcal{H}=\mathcal{H}_{\text {intra }}+\mathcal{H}_{\text {inter }}+\mathcal{H}_{\mathrm{DM}}$,

where

$$
\begin{aligned}
\mathcal{H}_{\text {intra }}= & J \sum_{j=1}^{N}\left(\boldsymbol{S}_{j, 1} \cdot \boldsymbol{S}_{j, 2}+\boldsymbol{S}_{j+\frac{1}{2}, 1} \cdot \boldsymbol{S}_{j+\frac{1}{2}, 2}\right),(2) \\
\mathcal{H}_{\text {inter }}= & J^{\prime} \sum_{j=1}^{N}\left\{\left(\boldsymbol{S}_{j, 1}+\boldsymbol{S}_{j, 2}\right) \cdot \boldsymbol{S}_{j+\frac{1}{2}, 1}\right. \\
& \left.+\boldsymbol{S}_{j+\frac{1}{2}, 2} \cdot\left(\boldsymbol{S}_{j+1,1}+\boldsymbol{S}_{j+1,2}\right)\right\} \\
\mathcal{H}_{\mathrm{DM}}= & \boldsymbol{D} \cdot \sum_{j=1}^{N}\left(\boldsymbol{S}_{j+\frac{1}{2}, 1} \times \boldsymbol{S}_{j, 1}+\boldsymbol{S}_{j, 1} \times \boldsymbol{S}_{j-\frac{1}{2}, 2}\right. \\
& \left.+\boldsymbol{S}_{j-\frac{1}{2}, 2} \times \boldsymbol{S}_{j, 2}+\boldsymbol{S}_{j, 2} \times \boldsymbol{S}_{j+\frac{1}{2}, 1}\right) .
\end{aligned}
$$

Here $N$ is the number of unit cells in the system, as shown by a broken rectangle in Fig. 1. The unit cell includes two dimers along vertical and horizontal direction, which are designated by the index, $j$ and $j+\frac{1}{2}$, respectively. $\boldsymbol{S}_{j, \alpha}(j=1,2, \cdots, N$ and $\alpha=$ $1,2)$ denotes a spin- $1 / 2$ operator on $\alpha$-spin in $j$ th dimer. $J$ and $J^{\prime}$ severally indicate the exchange coupling in intra-dimer and in inter-dimer. Due to the structure of system, the DM exchange interaction, $\boldsymbol{D}$, exists only on inter-dimer bonds and has only a component perpendicular to two kinds of dimer in the unit cell. The periodic boundary condition is imposed to the system.

\section{Ground state and low-energy excitations}

In this section, let us discuss the ground state and low-energy excitations of the 1D orthogonal dimer model with the DM interaction. We can expect that the ground state is in the dimer phase

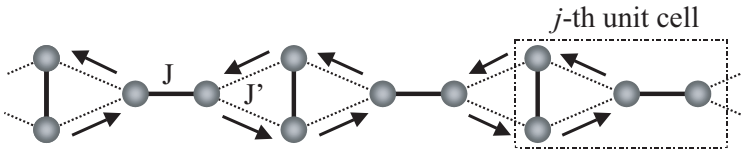

Fig. 1. The one-dimensional orthogonal-dimer model with the DM interaction. The thick and broken lines indicate intra- and inter-dimer interactions, respectively. The arrows on inter-dimer show the order of spins in the expression of the DM interaction $\boldsymbol{D} \cdot\left(\boldsymbol{S}_{i, \alpha} \times \boldsymbol{S}_{j, \beta}\right)$, that is $(i, \alpha) \rightarrow(j, \beta)$. The unit cell includes a vertical and horizontal dimer. The former dimers are at $j$-site and the latter at $\left(j+\frac{1}{2}\right)$-site.

in the limit of strong intra-dimer coupling $(J \gg$ $\left.J^{\prime},|\boldsymbol{D}|\right)$, even when the DM interaction is switched on the isotropic system. Therefore, it is reasonable to treat the intra-dimer Hamiltonian (2) as an unperturbated one and the others as perturbation.

The inter-dimer interaction $J^{\prime}$ creates two adjacent triplets from a pair of a singlet and triplet and vice versa, and besides shows scatterings between two triplets. The DM interaction not only causes the former process but also creates or annihilates two adjacent singlets. Therefore the DM interaction can play a crucial role in the ground state and the low-energy excitations in the dimer phase.

First, we discuss the ground-state energy of Hamiltonian (1). In the absence of the DM interaction, the ground state is exactly represented by a direct product of singlets and its energy is given as $\epsilon_{0} \equiv E_{0} / J=-\frac{3}{2} N$.

On the other hands, the ground-state energy of total Hamiltonian (1) is estimated as

$\frac{E_{g}}{J}=\epsilon_{0}-\frac{N}{2}\left(\frac{D}{J}\right)^{2}-\frac{N}{8} \frac{J^{\prime}}{J}\left(\frac{D}{J}\right)^{2}$.

from the perturbation expansion up to the third order in $J^{\prime} / J$ and $D / J$. The result means that the ground state cannot be exactly described by the direct product of singlets owing to the DM interaction.

Next, we argue the low-energy excitations in the system. Since the ground state belongs to the dimer phase in the region of strong- $J$, the lowest excited states will be well described by

$$
|M, k\rangle_{\mathrm{ver}} \equiv t_{M, k}^{\mathrm{ver} \dagger}|0\rangle
$$




$$
\begin{aligned}
& =\frac{1}{\sqrt{N}} \sum_{j=1}^{N} e^{i k j}\left|s_{1} \cdots t_{M, j} \cdots s_{N+\frac{1}{2}}\right\rangle, \\
& |M, k\rangle_{\text {hor }} \equiv t_{M, k}^{\text {hor }}|0\rangle \\
& =\frac{1}{\sqrt{N}} \sum_{j=1}^{N} e^{i k\left(j+\frac{1}{2}\right)}\left|s_{1} \cdots t_{M, j+\frac{1}{2}} \cdots s_{N+\frac{1}{2}}\right\rangle .
\end{aligned}
$$

Here, $M(=0, \pm 1)$ and $k$ are the total magnetization and the wave number, respectivery. $s_{j}$ and $t_{M, j}$ in ket severally denote a singlet and a triplet with $M$ at $j$-site and, $t_{M, k}^{\mathrm{ver} \dagger}\left(t_{M, k}^{\mathrm{hor} \dagger}\right)$ is defined as an operator to create a triplet propagating on vertical (horizontal) dimers. By using two states of Eqs. (6) and (7), the Hamiltonian (1) is projected on following $(2 \times 2)$-matrix:

$\mathcal{H}_{M}(k)=\boldsymbol{v}_{M}^{\dagger}(k) \boldsymbol{A}_{M}(k) \boldsymbol{v}_{M}(k)$

where

$\boldsymbol{A}_{M}(k) \equiv\left[\begin{array}{ll}a_{11, M} & a_{12, M} \\ a_{21, M} & a_{22, M}\end{array}\right], \boldsymbol{v}_{M}(k) \equiv\left[\begin{array}{c}t_{M, k}^{\mathrm{ver}} \\ t_{M, k}^{\mathrm{hor}}\end{array}\right]$

The Eq. (8) for $M=0$ has no off-diagonal elements within perturbation up to the third order. Therefore the excitation energies for $M=0$ are given by

$$
\begin{aligned}
\frac{E_{0}^{\mathrm{ver}}(k)}{J}= & \epsilon_{0}+1-\frac{N-1}{2}\left(\frac{D}{J}\right)^{2}-\left(\frac{J^{\prime}}{J}\right)^{2} \\
& -\frac{N-1}{8} \frac{J^{\prime}}{J}\left(\frac{D}{J}\right)^{2}-\frac{1}{2}\left(\frac{J^{\prime}}{J}\right)^{3} \\
\frac{E_{0}^{\mathrm{hor}}(k)}{J}= & \epsilon_{0}+1-\frac{N+1}{2}\left(\frac{D}{J}\right)^{2} \\
& -\left(\frac{N+3}{8}+\cos k\right) \frac{J^{\prime}}{J}\left(\frac{D}{J}\right)^{2}
\end{aligned}
$$

In contrast to the $2 \mathrm{D}$ orthogonal dimer model, two excitation energies, $E_{0}^{\text {ver }}(k)$ and $E_{0}^{\text {hor }}(k)$, split in the case of $1 \mathrm{D}$ system. It is also interesting to note that the curvature of $E_{0}^{\text {hor }}(k)$ appears in the third ordered correction in Eq. (11).

On the other hand, the projected Hamiltonian with $M= \pm 1$ has an off-diagonal element. The perturbation calculation up to the third order leads to the matrix:

$$
\boldsymbol{A}_{ \pm 1}=\left[\begin{array}{cc}
a+b_{k} & -i d_{k} \\
i d_{k} & a+c
\end{array}\right]
$$

where

$$
\begin{aligned}
a= & \epsilon_{0}+1-\frac{N-1}{2}\left(\frac{D}{J}\right)^{2}-\left(\frac{N}{8}-\frac{3}{16}\right) \frac{J^{\prime}}{J}\left(\frac{D}{J}\right)^{2}, \\
b_{k}= & -\left(\frac{J^{\prime}}{J}\right)^{2}-\frac{1}{4}\left(\frac{D}{J}\right)^{2} \cos k-\frac{1}{2}\left(\frac{J^{\prime}}{J}\right)^{3} \\
& -\frac{1}{2}(1+\cos k) \frac{J^{\prime}}{J}\left(\frac{D}{J}\right)^{2}, \\
c= & -\frac{1}{2}\left(\frac{D}{J}\right)^{2}, \\
d_{k}= & \left(\frac{D}{J}+\frac{J^{\prime} D}{2 J^{2}}-\frac{J^{\prime 2} D}{4 J^{3}}-\frac{J^{\prime} D^{2}}{4 J^{3}}+\frac{D^{3}}{2 J^{3}}\right) \cos \frac{k}{2} .
\end{aligned}
$$

By diagonalizing Eq. (12), the excitation energies with $M= \pm 1$ are obtained as

$\frac{E_{ \pm 1}^{( \pm)}(k)}{J}=a+\frac{b_{k}+c}{2} \pm \sqrt{d_{k}^{2}+\frac{\left(b_{k}-c\right)^{2}}{4}}$.

The curvature of $E_{ \pm 1}^{( \pm)}(k)$ is dominant by the first ordered correction with regard to $D / J$ in the offdiagonal element $d_{k}$. The correction derives from the scattering between a singlet and a triplet with $M= \pm 1$ due to the DM interaction.

Subtracting the ground-state energy of Eq. (5) from excited-state energies of Eq. (10), (11), and (13), the low-energy dispersions, $\omega_{k, M} \equiv\left(E_{M}(k)-\right.$ $\left.E_{g}(k)\right) / J$, are estimated as

$$
\begin{aligned}
\omega_{0}^{\text {ver }}(k)= & 1+\frac{1}{2}\left(\frac{D}{J}\right)^{2}-\left(\frac{J^{\prime}}{J}\right)^{2} \\
& +\frac{1}{8} \frac{J^{\prime}}{J}\left(\frac{D}{J}\right)^{2}-\frac{1}{2}\left(\frac{J^{\prime}}{J}\right)^{3}, \\
\omega_{0}^{\text {hor }}(k)= & 1-\frac{1}{2}\left(\frac{D}{J}\right)^{2}-\left(\frac{3}{8}+\cos k\right) \frac{J^{\prime}}{J}\left(\frac{D}{J}\right)^{2}, \\
\omega_{ \pm 1}^{( \pm)}(k)= & +\frac{1}{2}\left(\frac{D}{J}\right)^{2}-\left(\frac{J^{\prime}}{J}\right)^{2}+\frac{3}{16} \frac{J^{\prime}}{J}\left(\frac{D}{J}\right)^{2} \\
& +\frac{b_{k}+c}{2} \pm \sqrt{d_{k}^{2}+\frac{\left(b_{k}-c\right)^{2}}{4}} .
\end{aligned}
$$




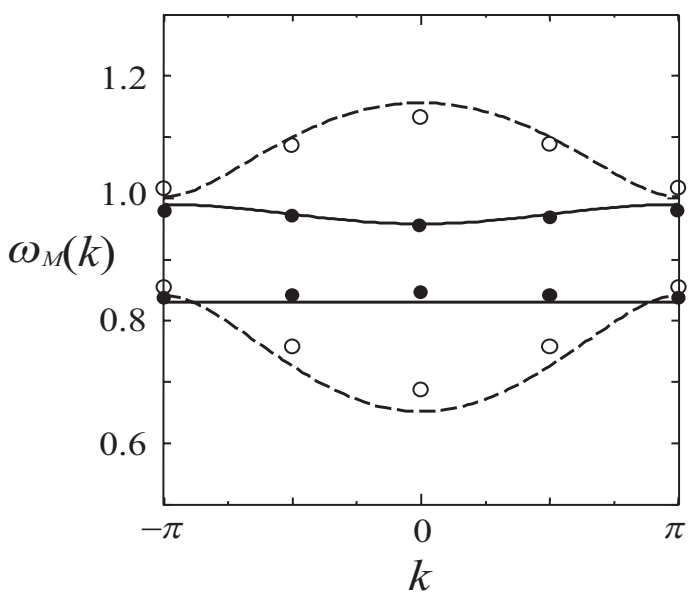

Fig. 2. The low-energy dispersions for $J^{\prime} / J=0.4$ and $D / J=0.2$. The solid and dotted curves are of $M=0$ and $M= \pm 1$, respectively. The full (open) circles indicates the excitation energies for $M=0(M= \pm 1)$ calculated by numerical diagonalizations.

Figure 2 shows the low-energy dispersions for $J^{\prime} / J=0.4$ and $D / J=0.2$. The low energy spectra $\omega_{0}^{\text {ver }}$ and $\omega_{0}^{\text {hor }}$ are severally represented by the lower and upper solid lines, and then the upper and lower dotted lines denote $\omega_{ \pm 1}^{(+)}$and $\omega_{ \pm 1}^{(-)}$in Eqs. (16). The full and open circles represent the low-energy spectra with $M=0$ and $M= \pm 1$. The perturbation theory is in agreement with the numerical diagonalization, as to low-energy excitations. The dispersions with same $M$ are not degenerate, which happens even if the DM interaction is not taken account of. Therefore the inter-dime coupling $J^{\prime}$ is also important for splitting of the low-energy dispersions with same $M$. This is because the parity on the vertical dimer is conserved in the one-dimension system without the DM interaction. The DM interaction not only splits into branches with $M=0$ and $M= \pm 1$, but also makes triplets move more easily.

\section{Summary}

We investigated the low-energy excitations in the 1D orthogonal-dimer model with the DM interaction using the perturbation theory and numerical exact-diagonalization method. The DM interaction allows a triplet to propagate in singlet sea as seen in the 2D system [11], while the triplet is localized on vertical or horizontal dimer in the absence of the DM interaction. This curvature effect happens in a two-dimensional orthogonal-dimer model, but the splitting of spectra reflects a stringent constraint for the motion of a triplet due to one dimensionality as well as the DM interaction.

Acknowledgements We gratefully acknowledge helpful discussions and comments with Tetsuro Nikuni, Masaaki Nakamura, and Takahiro Yamamoto on several points in this work. We would also like to thank Kôichirô Ide for valuable advice on numerical techniques.

\section{References}

[1] H. Kageyama, et al. : Phys. Rev. Lett. 82 (1999) 3168.

[2] K. Onizuka, et al. : J. Phys. Soc. Jpn. 69 (2000) 1016.

[3] S. Miyahara and K. Ueda: Phys. Rev. Lett. 87 (2001) 3701.

[4] B. S. Shastry and B. Sutherland: Physica B 108 (1981) 1089.

[5] H. Nojiri, et al.: J. Phys. Soc. Jpn. 68 (1999) 2906.

[6] I. Dzyaloshinski: J. Phys. Chem. Solids 4 (1958) 241; T. Moriya: Phys. Rev. 120 (1960) 91.

[7] O. Cépas, et al. : Phys. Rev. Lett. 87 (2001) 167205.

[8] A. Koga, K. Okunishi and N. Kawakami: Phys. Rev. B. 62 (2000) 5558

[9] N. B. Ivanov and J. Richter : Phys. Lett. A 232 (1997) 308.

[10] J. Richter, N. B. Ivanov and J. Shulenburg : Phys. Rev. Lett. 88 (2002) 201601.

[11] S. Miyahara and K. Ueda: J. Phys. Soc. Jpn. (Suppl.) B 70 (2001) 180. 